\title{
Scrutinizing the Stability and Exploring the Dependence of Thermoelectric Properties on Band Structure of $3 d$ Metal-Based Double Perovskites: An ab-initio Analysis
}

Shabir Ahmad Mir ( $\nabla$ mirshabir7500@gmail.com )

Jiwaji University

Dinesh C. Gupta

Jiwaji University

\section{Research Article}

Keywords: Ba2FeNiO6, DFT, thermoelectric technology, energy

Posted Date: February 19th, 2021

DOI: https://doi.org/10.21203/rs.3.rs-206716/v1

License: (c) (i) This work is licensed under a Creative Commons Attribution 4.0 International License.

Read Full License 


\section{Abstract}

Through the conventional DFT computation, we have designed new oxide double perovskites Ba2BNiO6 $(\mathrm{B}=\mathrm{Fe}$ and $\mathrm{Co})$. The structural and thermodynamic stabilities are defined by optimizing the crystal energy and determination of tolerance factor and cohesive energies. Thereafter, at the optimized lattice constant, we have explored the different physical properties. The GGA+mBJ electronic band-structure depicts the semiconducting nature for Ba2CoNiO6 while half-metallic with $100 \%$ spin polarization for $\mathrm{Ba} 2 \mathrm{FeNiO6}$. The origin of such a diverse band profile upon changing $\mathrm{Fe}$ to $\mathrm{Co}$ is explained with the help of the orbital diagram and exchange interaction. The eg-eg interaction is strong in these perovskites compared to eg-t2g and $\mathrm{t} 2 \mathrm{~g}-\mathrm{t} 2 \mathrm{~g}$ hybridization. The strong exchange interaction among eg states via 0-p states happens because the B-O-Ni angle is strictly $180^{\circ}$. Furthermore, due to the narrow bandgaps, we have explored the transport properties to express the applicability of these materials towards thermoelectric technology. Also, herein we have investigated the dependency of transport properties on band profile. The semiconducting nature in $\mathrm{Ba} 2 \mathrm{CoNiO6}$ results in a high $\mathrm{ZT} \sim 0.8$ at room temperature makes it suitable for energy restoration.

\section{Introduction}

With the passage of time and advancement in experimental techniques new materials that are multifunctional and could be used in current and futuristic technologies are being discovered. The discovery of the new novel materials retaining the promising capabilities to meet the challenges for new technological applications is at new heights. In the last year, room temperature superconductivity and solar cells with $47.1 \%$ conversion efficiency at lab scale were successfully characterized $[1,2]$. Besides the experimental synthesis of the novel materials, density functional theory (DFT) based simulations have been verified to be one of the most accurate and efficient methods to find and explore new materials $[3,4,5]$. With the combined experimental and DFT-methods, unusual temperature dependence with ZT 470 at around 350K has been reported [6]. New members of a series of families are being intensively designed through DFT simulations by establishing their stabilities and computation of the electronic structure along with other physical properties $[7,8,9,10]$. The DFT-investigation is quite helpful in predicting the nature of the materials under extreme conditions with economic-facile methods. So, DFT examination can be considered to be the first step to define stability and explore properties of any material in a quick process before its experimental synthesis.

In the present era, perovskites represent one of the noble families of materials being prestigious for the scientific community. Oxide double perovskites (DPs) are the quaternary metal oxides with a chemical composition of $\mathrm{A}_{2} \mathrm{BB} \mathrm{O}_{6}$. The A-site occupants are mostly alkali-earth metal but any other metal that can exist in the +3 or +2 -oxidation state with coordination number 12 can occupy the site. The most occurring oxidation states found for double perovskites are $\mathrm{A}_{2}{ }^{+2}(\mathrm{BB} \square)^{+8} \mathrm{O}_{6}{ }^{-2}$ and $\mathrm{A}_{2}{ }^{+3}(\mathrm{BB} \square)^{+6} \mathrm{O}_{6}{ }^{-2}$ but rarely occur in $\mathrm{A}_{2}{ }^{+1}(\mathrm{BB} \square)^{+10} \mathrm{O}_{6}{ }^{-2}[11,12,13]$. The $\mathrm{B}$ and $\mathrm{B} \otimes$ constituents are mostly transition/inner transition metals. The ionic character makes these materials suitable for solid-state ionic batteries [14]. Besides this double 
perovskite materials are known to be multifunctional showing good oxidation resistance, high curie temperature, large spin polarization effect, a high figure of merit, and other fascinating properties $[15,16,17,18]$. These features make double perovskites apposite for many advanced technologies. In this paper, we have designed two new DPs, $\mathrm{Ba}_{2} \mathrm{BNiO}_{6}(\mathrm{M}=\mathrm{Fe}$ and $\mathrm{Co})$, having a $3 d-3 d$ combination of transition metals $\mathrm{Fe}^{+5}\left(3 \mathrm{~d}^{3}, \mathrm{t}_{2 \mathrm{~g}}{ }^{3} \mathrm{e}_{\mathrm{g}}{ }^{0}\right) / \mathrm{Co}^{+5}\left(3 \mathrm{~d}^{4}, \mathrm{t}_{2 \mathrm{~g}}{ }^{3} \mathrm{eg}^{1}\right)$ and $\mathrm{Ni}^{3+}\left(3 \mathrm{~d}^{7}, \mathrm{t}_{2 \mathrm{~g}}{ }^{6} \mathrm{eg}^{1}\right)$. The constituents of these materials are present in quantum quantity in the earth's crust. Moreover, the doped form of these double perovskites and similar other perovskites has been experimentally reported. Recently, Shen et al. have synthesized $\mathrm{Ba}_{2} \mathrm{Ni}_{\mathrm{x}} \mathrm{Co}_{2-\mathrm{x}} \mathrm{O}_{6}(\mathrm{x}=0.15,0.35,0.55,0.75)$ showing good regeneration ability [19]. $\mathrm{Ba}_{2} \mathrm{FeCoO}_{6-}$ ${ }_{\delta}$ oxygen-deficient double perovskite has been reported to be a potential electrode material for super capacitive purposes [20]. However, by the computation means, $\mathrm{Ba}_{2} \mathrm{FeMnO}_{6}$ has been reported to show half-metallic nature with a high magnetic moment of $6 \mu_{B}$ with a large thermopower [21]. $A_{2} \mathrm{MnTaO}_{6}(A=$ $\mathrm{Sr}, \mathrm{Ba})$ are found to be cubic half-metallic ferromagnetic materials $[22,23]$. In our previous studies, we have found transition metal-based perovskites $\mathrm{La}_{2} \mathrm{CuMnO}_{6}$ and $\mathrm{Ba}_{2} \mathrm{CoUO}_{6}$ are ferromagnetic half-metals while $\mathrm{Ba}_{2} \mathrm{NiUO}_{6}$ is a ferromagnetic semiconductor $[24,25]$.

In the transition metal-based perovskites, the $d$ - $d$ electronic interaction is so strong leading to localize the electron orbital and spin moments [26]. The B-O-B' bond angle features the type of exchange interaction [27]. The B-B' interactions of double perovskites can be are gradually altered by carefully manipulating the $B-O-B^{\prime}$ bond angle to obtain the desired properties $[28,29]$. If both constituents (B and $\left.B^{\prime}\right)$ are magnetic, the magneto-electronic structure of such configuration is governed by $\mathrm{B}-\mathrm{O}-\mathrm{B}^{\prime}$ next neighbor $\mathrm{B}-\mathrm{B}^{\prime}$ interaction. However, if one of the transition-site ions is non-magnetic ( $B^{\prime}$ say), the properties are defined by long-distance next-next-nearest neighbor (B-B) exchange interactions Therefore, the choice of $d$-atoms having different electron occupancies largely affects the magneto-electronic thereby transport and mechanical properties of the DP's.

\section{Results And Discussions}

The obtained results of the study and the discussion over the results are presented below under different sections

\section{Structural and Thermodynamic Stabilities}

Double perovskites $\mathrm{A}_{2} \mathrm{BB} \square \mathrm{O}_{6}$ are a modified form of simple perovskites $\mathrm{ABO}_{3}$ by replacing exactly half of the B-cations with different B囚-cation [12]. Ideal simple perovskites are most stable in $\mathrm{Pm}-3 \mathrm{~m}$ cubic structure given in Fig. S1 (Supplementary Information). However, the transition from simple perovskite to double perovskite changes the prototype structure from Pm-3m to Fm-3m shown in Fig. S1. Also, the lattice constant in DPs is almost double of simple perovskites. The $\mathrm{B}$ and $\mathrm{B} \otimes$ cations in $\mathrm{Fm}-3 \mathrm{~m}$ structure are mostly ordered; occupy alternative sites or even can be layer-wise ordered. Like in the simple perovskites, the mismatch in the sizes of the constituents can distort the structure. Therefore, tolerance factor ' $t$ ' an empirical relation from ionic radii of constituents is most widely used to predict the structure 
of new double perovskites [25]. If ' $\mathrm{t}$ ' is in the range of $0.9-1$, the cation sizes are perfect for the ideal structure. However, when $\mathrm{t}<0.9$ and $\mathrm{t}>1$ the constituents are in an under bonded state, therefore $\mathrm{BO}_{6}$ and $\mathrm{B} \square \mathrm{O}_{6}$ octahedra are distorted. The calculated t-factor is presented in Table 1 suggests the stability of titled perovskites in $\mathrm{Fm}-3 \mathrm{~m}$ structure. To be more convinced about the stability of $\mathrm{Ba}_{2} \mathrm{BNiO}_{6}(\mathrm{M}=\mathrm{Fe}$ and $\mathrm{Co}$ ) in a cubic structure, we have carried out optimization via spin-polarized and non-spin polarized calculations. The Brich-Murganian equation is used to make fit from energy-volume data and predict the optimized parameters $[30,31]$.

$$
E(V)=E_{0}+\frac{9 B_{0} V_{0}}{16}\left\{\left[\left(\frac{V_{0}}{V}\right)^{2 / 3}-1\right] B_{0}^{\prime}+\left[\left(\frac{V_{0}}{V}\right)^{2 / 3}-1\right]^{2}\left[6-4\left(\frac{V_{0}}{V}\right)^{2 / 3}\right]\right\}
$$

Here, $E_{0}, V_{0}, B_{0}$, and $B \square_{0}$ represent energy, volume, bulk modulus, and pressure derivative of $B_{0}$ respectively in the stress-free state. The optimization curves are presented in Fig. 1 and the optimized parameters are reported in Table 1. The parabolic character of the curves authenticates the stability in a cubic structure. On comparing the energy of the magnetic and non-magnetic phase it is evident that the magnetic phase is most stable.

Table 1: Optimized parameters of $\mathrm{Ba}_{2} \mathrm{BNiO}_{6}$ double perovskites

\begin{tabular}{|lllllll|}
\hline Material & $\begin{array}{l}\text { Tolerance } \\
\text { factor }()\end{array}$ & $\begin{array}{l}\text { Lattice } \\
\text { Constant } \\
\text { a (̊) }\end{array}$ & $\begin{array}{l}\text { Bulk } \\
\text { Modulus } \\
\mathrm{B}(\mathrm{GPa})\end{array}$ & $\begin{array}{l}\text { Pressure derivative } \\
\text { of bulk modulus } \\
\mathrm{B}^{\prime}(\mathrm{GPa})\end{array}$ & $\begin{array}{l}\text { Total } \\
\text { energy E } \\
(\mathrm{Ry})\end{array}$ & $\begin{array}{l}\text { Cohesive } \\
\text { energy }\end{array}$ \\
\hline $\mathrm{Ba}_{2} \mathrm{FeNiO}_{6}$ & 0.93 & 7.90 & 130.11 & 4.93 & -38793.58 & 12.65 \\
\hline $\mathrm{Ba}_{2} \mathrm{CoNiO}_{6}$ & 0.95 & 7.89 & 132.11 & 4.79 & -39289.46 & 13.32 \\
\hline $\mathrm{Ba}_{2} \mathrm{FeMnO}_{6}{ }^{[21]}$ & 0.99 & 7.97 & 148.17 & 5.05 & & \\
\hline
\end{tabular}

The thermodynamic stability of these compounds is predicted by computing the cohesive energy $\left(\mathrm{E}_{\mathrm{C}}\right)$. The cohesive energy of a compound is defined by the difference between the total cell energy calculated at the equilibrium lattice constant, and the atomic energy calculated for the fundamental state configuration of $\mathrm{Ba}, \mathrm{B}, \mathrm{Ni}$, and $\mathrm{O}$ according to the following formula [32]:

$$
E_{C}=E_{\text {total }}^{\text {Bamio6 }}-\left\{2 E_{\text {aom }}^{B a}+E_{\text {atom }}^{B}+E_{\text {atom }}^{N i}+6 E_{\text {atom }}^{O}\right\}
$$

All these energies are computed by using the GGA-PBE approximation and the obtained values are given in Table 1. Positive values of magnitude 12.65 Ry and 13.52 Ry for FeNi and CoNi-perovskites support the stability of the materials. Besides cohesive energy, we have computed other thermodynamic properties and plotted their variation with temperature are shown in Fig. S2. Specific heat $\left(C_{v}\right)$ is the amount of 
energy required to raise the temperature of that material by one degree. Therefore, specific heat represents the energy that can be stored in a material for a given temperature difference. If the temperature of that material is lowered back to the initial temperature the specific heat is converted back to energy. So, the higher the heat capacity more could be the energy store and likely material could be used as an efficient regenerator. The specific heat plot of the titled materials is given in Fig. S2(a). The $C_{v^{-}}$ variation with temperature indicates at low-temperature $C_{v}$ follows $\mathrm{T}^{3}$ law only longwave phonon are excited in this range [33]. However, towards high temperature, all the phonons are thermally excited and $C_{v}$ tends to Dulong limit value $3 \mathrm{nR}, \mathrm{R}$ is gas constant [34]. Moreover, the plot of $C_{v}$, reflects there is no structural phase transition in the entire temperature range. The other thermodynamic parameters like Grüneisen parameter $(Y)$ and Debye temperature $\left(\theta_{D}\right)$ are discussed in the Supplementary Information.

\section{Electronic and Magnetic Behaviour}

The band structure of $\mathrm{Ba}_{2} \mathrm{BNiO}_{6}$ double perovskites obtained by GGA is represented by Fig. S3. The GGA band structure indicates the metallic character of these perovskites as the Fermi level passes through the bands. However, by incorporating $\mathrm{mBJ}$ potential to GGA, the band structure changes effectively. The change in band structure is obvious because GGA underestimates the exchange-correlation potential and incorporation of $\mathrm{mBJ}$ potential sophisticatedly improves the results [34,35]. The GGA+mBJ band profile represented in Fig. 2, reveals $\mathrm{Ba}_{2} \mathrm{CoNiO}_{6}$ is semiconducting in both spin channels. While $\mathrm{Ba}_{2} \mathrm{FeNiO}_{6}$ is half-metallic, designates metallic character in the spin-up channel and poses semiconducting behavior in the spin-down channel. The half-metallic character of $\mathrm{Ba}_{2} \mathrm{FeNiO}_{6}$ thereby suggests $100 \%$ spin polarization around the Fermi level.

To further discuss the electronic properties and elucidate the band structure of the materials we have analyzed the distribution of energy states over an energy range of $-8 \mathrm{eV}$ to $6 \mathrm{eV}$. The total density of states (TDOS) obtained from GGA and GGA+mBJ methods are given in Fig. 3. With the implication of $\mathrm{mBJ}$, the energy states sweep away and open the gap at the Fermi level. Moreover, the atomic projected density of the states is given by Fig. S4, the energy states of interest are Fe- $d$, Co- $d$, Ni- $d$, and 0- $p$. In FeNibased perovskites the distribution of states is as; In the spin-up channel, Fe- $t_{2 g}$ and Ni- $t_{2 g}$ states are filled happen below Fermi level but $\mathrm{Fe}-e_{g}$ and $\mathrm{Ni}-e_{g}$ state strongly hybridize with the 0- $p$ states occupy the Fermi level. While in the spin-down channel, Ni- $t_{2 g}$ states are filled but Fe- $t_{2 g}$ states are empty contribute towards conduction band formation as can be seen from Fig. 4. The Fe $\left(e_{g}\right)-\mathrm{O}(p)-\mathrm{Ni}\left(e_{g}\right)$ states even in the spin-down state strongly hydride contributes one peak below fermi level while one above it. Also, in the $\mathrm{Ba}_{2} \mathrm{CoNiO}_{6}$ system, the $e_{g}$-states $\left\{\mathrm{Co}\left(e_{g}\right)-\mathrm{O}(p)-\mathrm{Ni}\left(e_{g}\right)\right\}$ strongly hybridize via 0- $p$ states constitute two subbands one in valance band and others in conduction. The Co- $t_{2 g}$ states are filled in the spin-up state and empty in the down channel, while $\mathrm{Ni}-t_{2 g}$ states are filled for both channels. No states are occupying the Fermi level in both spin orientations reflecting the semi-conducting type nature of $\mathrm{Ba}_{2} \mathrm{CoNiO}_{6}$. The bandgap values are provided in Table 2 .

Table 2: Calculated band gap ( $\mathrm{E}_{\mathrm{g}}$ in eV) and Magnetic moment $\left(\mu_{B}\right)$ of $\mathrm{Ba}_{2} \mathrm{MNiO}_{6}$ materials 


\begin{tabular}{|c|c|c|c|c|c|c|c|}
\hline \multirow[t]{3}{*}{ Material } & \multicolumn{4}{|c|}{ Bandgap } & \multicolumn{3}{|c|}{ Magnetic Moment } \\
\hline & \multicolumn{2}{|c|}{$\mathrm{Ba}_{2} \mathrm{FeNiO}_{6}$} & \multicolumn{2}{|c|}{$\mathrm{Ba}_{2} \mathrm{CoNiO}_{6}$} & \multirow[t]{2}{*}{$\mathrm{Ba}_{2} \mathrm{FeNiO}_{6}$} & \multirow[t]{2}{*}{$\mathrm{Ba}_{2} \mathrm{CoNiO}_{6}$} & \multirow[t]{2}{*}{$\mathrm{Ba}_{2} \mathrm{FeMnO}_{6}^{[21]}$} \\
\hline & $\uparrow$ & $\downarrow$ & $\uparrow$ & $\downarrow$ & & & \\
\hline GGA & - & - & - & - & 4.59 & 5.72 & 7.0 \\
\hline GGA+mBJ & - & 1.66 & 0.11 & 1.22 & 4.0 & 5.0 & \\
\hline
\end{tabular}

\section{Origin of semiconducting gap}

The illustration of the driving mechanism for the understanding role of $e_{g}$-states in the origin of such a band profile is given in Fig $\mathbf{5}(\mathbf{a}, \mathbf{b}, \mathbf{c})$. The well-known behavior of the $d$-states in the octahedra field is that they split into two separate degenerate sets $t_{2 g}$ and $e_{g}$ sub-sets. The $3 d$-atoms in $\mathrm{Ba}_{2} \mathrm{BNiO}_{6}$ configuration occupy alternative sites that are bonded via O-atoms. The B-O-Ni bond angle for the present set of materials is $180^{\circ}$. Therefore, the $e_{g}$-states of $\mathrm{Fe}$ and $\mathrm{Ni}$ (similarly $\mathrm{Co}$ and $\mathrm{Ni}$ ) in their respective materials are linearly along the axes. However, the $t_{2 g}$ states are in the plane don't orient directly towards the orbitals of the nearest neighbor so mostly don't take part in the hybridization, hybridize feebly in a lateral way with $\mathrm{t}_{2 \mathrm{~g}}$ and $\mathrm{e}_{\mathrm{g}}$-states of another atom. The hybridization between d-states of transition atom with 0p can also be visualized from charge density distribution shown in Fig. 5(b). The B and Ni-atoms are covalently bonded with $\mathrm{O}$-atom. While Ba-O bonds show ionic bonding.

The orbital splitting and formation of bonding and anti-bonding $e_{\mathrm{g}}$-states are represented via Fig. $\mathbf{5}$ (c). The nominal valance states of the present set of double perovskites are $\mathrm{Ba}_{2}{ }^{+2} \mathrm{~B}^{+5} \mathrm{Ni}^{+3} \mathrm{O}^{-2}$, maintaining the charge stability. The $\mathrm{B}^{+5}$ and $\mathrm{Ni}^{+3}$ electrons are most dominant in characterizing the electronic band structure. The six electrons out of seven in $\mathrm{Ni}^{3+}$ for both perovskites fill $t_{2 g}$-states forming a low spin state. The last electron of $\mathrm{Ni}^{3+}$ enters hybridized $e_{g}-e_{g}$ bonding states. Now in $\mathrm{Ba}_{2} \mathrm{FeNiO}_{6}$, the three unpaired electrons in $\mathrm{Fe}^{+5}$ half fill $t_{2 g}$, i.e., $t_{2 g}$ states are filled in spin-up channel localize them in the valance band. However, a single electron in hybridized bonding states of $(\mathrm{Fe}) e_{g}-(\mathrm{Ni}) e_{g}$ bonding states fills them partially and make them reside at the Fermi level of the spin-up channel as can be seen from the Fig. 5(c). Likewise, $\mathrm{Co}^{5+}$ in $\mathrm{Ba}_{2} \mathrm{CoNiO}_{6}$ also forms a high spin state, three electrons out of four fill spin-up $t_{2 g}$ state. While the last (fourth) electron and one from $\mathrm{Ni}^{3+}$, half fill the hybridized bonding (Co) $e_{g^{-}}$ (Ni) $e_{g}$ states, bring them down to the valance band in the spin-up channel and open the gap at Fermi level. In the spin-down channel of both materials, only $\mathrm{Ni}-t_{2 g}$ states are filled lie in the valence band, while all other $d$-states that are empty reside in the CB with a gap at the Fermi level. The overall number of unpaired electrons in FeNi- and CoNi-perovskites is 4 and 5, due to which the magnetic respective materials are $4 \mu_{B}$ and $5 \mu_{B}$ following the Slater-Pauling rule [36].

\section{Thermoelectric Properties}


The transport character of the materials is directly linked to the electronic band structure. Ideally, the perfect thermoelectric material should possess a large thermopower like an insulator and low resistivity like a metal, which is almost impossible to attain practically. However, low bandgap degenerate semiconductors enjoy the sweet spot among the thermoelectric applicable materials. Therefore, such materials are on the hunt for thermoelectric technology and are expected to show a good thermoelectric response. The thermoelectric response of $\mathrm{Ba}_{2} \mathrm{MNiO}_{6}$ materials has been recorded by analyzing the variation of various transport parameters with chemical potential at different temperatures. The dissimilar electronic filling in spin channels of magnetic materials suggests electrons in these spin channels experience different driving forces thereby exhibit variant scattering rates. The variation in the transport parameters viz. Seebeck coefficient, electronic conductivities is directly linked with the nature of the energy bands around the Fermi level. The variation in total Seebeck coefficient, total conductivities along with the figure of merit (ZT) is presented in Fig. 6 to Fig. 9. However, the electronic band profile of spin up and spin down channels are entirely different. So, it is naturals for titled materials to exhibit dissimilar variational behavior of transport parameters in spin-up and spin-down channels discussed in Supplementary Information. The resultant conduction and Seebeck coefficient are defined with the help of two current model. According to which: and ; where arrows designate up and down channels [37,38,39]. The total Seebeck coefficient of FeNi-based double perovskite has several kinks, presented in Fig. 6(a), nevertheless, the peaks values remain low. While on the other side CoNi- shows a high Seebeck coefficient with main peaks occurring on either side of the Fermi level at 0.00 Ry refer to Fig. 6(b). With temperature rise, the peak values decline rapidly, nevertheless, the peak location remains the same. On comparing the magnitude of $\mathrm{S}, \mathrm{Ba}_{2} \mathrm{CoNiO}_{6}$ shows higher thermopower than $\mathrm{Ba}_{2} \mathrm{FeNiO6}$. The is mostly because of the semiconducting nature in both spin channels of $\mathrm{Ba}_{2} \mathrm{CoNiO}_{6}$ compared to the metallic nature in one spin channel of $\mathrm{Ba}_{2} \mathrm{FeNiO}_{6}$. Thereby, confirming that magnitude of Seebeck is directly related to the behavior of energy levels close to the Fermi level. With the increase in temperature the peak values of $|S|$ decreases greatly. The decreasing behavior of $|S|$ can be credited to the smearing of energy bands.

The variation in the total electronic conductivity is presented in Fig. $7(\mathbf{a}, \mathbf{b})$. The variation in conductivity with temperature is gentle but with the chemical potential, it is steep. Corresponding to the forbidden gap in band structure conductivity would be zero, therefore vanishing conductivity at 0 Ry of CoNi-reflect the semiconducting nature. However, with the rise in temperature conductivity increases slightly at the Fermi level it is because of the smearing of energy bands. On the other side, although the band structure of FeNi-based perovskite reflects half-metallic nature, total conductivity depicts the behavior of the conductor. The conductivity peaks at the Fermi level are wholly contributed by spin up channel, which is demonstrated by plotting conductivity against the chemical potential of spin up and down channels separately shown in Supplementary Information. As the value of chemical potential changes, the Seebeck and conductivity coefficients at some places increase with temperature, and somewhere shows a decreasing trend. The behavior is expected because of the presence of pseudo gaps and fewer states at some energy values of the band structure. Howsoever, as the temperature increases, bands smear, and some states that were empty at $\mathrm{T}=\mathrm{OK}$ are now filled because electrons make the transition due to a gain in thermal energy. The highly populated DOS regions have low effective mass, thereby low Seebeck 
coefficient and high conductivity. As the band smearing happens the effective mass increase, therefore, Seebeck coefficient increase while conductivity decrease. The reverse is the case for the low DOS populated regions. The electronic thermal conductivity presented in Fig. 8(a, b) also demonstrates a similar kind of behavior against chemical potential variation. However, the thermal conductivity increases abruptly with temperature compared to electric conductivity. Also, FeNi- shows higher conducting capacity compared to CoNi-perovskite. It because of the half-metallic electronic profile.

The figure of merit (ZT) is the ultimate factor that characterizes the desirability of materials towards thermoelectric applicability. The variation in ZT with chemical potential at different temperatures is illustrated via Fig. $9(\mathbf{a}, \mathbf{b})$. The ZT-value of FeNi-based perovskite is very low, the highest value goes around 0.013 that too away from the Fermi level at $-0.12 \mathrm{Ry}$. Although $\mathrm{Ba}_{2} \mathrm{FeNiO}_{6}$ has $100 \%$ spin polarization at the Fermi level however the thermoelectric results are poor mostly because of high thermal conductivity and low Seebeck coefficient. The overall transport properties of $\mathrm{Ba}_{2} \mathrm{FeNiO}_{6}$ have the effectively dominant character of the metallic channel. The spin split ZT is shown in the Supplementary Information, wherefrom it can be seen spin-down channel of FeNi-based having semiconducting nature has ZT 1. However, CoNi-based perovskite offers significant peaks on either side of the Fermi level with prominent peaks having $\mathrm{ZT} \sim 0.8$ in closeness to the Fermi level. the high value of $\mathrm{ZT}$ in $\mathrm{Ba}_{2} \mathrm{CoNiO}_{6}$ can be credited to the semiconducting band profile. However, with the temperature rise, the peak values drop down because of the combined effects of the decrease in Seebeck value and an increase in thermal conductivity.

\section{Computation Methodology}

All the calculations in the present study have been carried out with the help of the Wein2k simulation code in its full potential formalism [40]. The ground-state electron density for the perovskites is obtained with help of the Kohn-Sham (K-S) equation; wherein the exchange and correlation interaction have been estimated by well-known generalized gradient approximation parameterized of Perdew, Burke, and Ernzerhof [41]. The more detailed information regarding the parameters fitted for the present study is mentioned in Supplementary Information. Moreover, we have facilitated GGA by the modified BeckeJohnson (mBJ) potential to be more accurate [42]. The transport efficiency is computed with the help of Boltztrap code [43], wherein the Boltzmann equation is solved under the approximation of constant relaxation time $(\tau)$. The relaxation time is a variable parameter, the magnitude of ' $\tau$ ' undoubtedly affects the transport features. However, if the variations in ' $\tau$ ' are gentle on the energy scale, then constant relaxation time approximation works efficiently $[44,45]$. The thermodynamic properties are obtained with the help of Gibbs2 package integrated with Wien2k code [46].

\section{Conclusion}

In the presented work, the structural stability along with electronic and transported properties are investigated using the FP-LAPW scheme with GGA and GGA+mBJ approximations. The mBJ functional 
employed is found to produce to half-metallic charter for $\mathrm{Ba}_{2} \mathrm{FeNiO}_{6}$ and semiconducting nature for $\mathrm{Ba}_{2} \mathrm{CoNiO}_{6}$. The possible strong exchange interaction happens between the $e_{g}$ of $\mathrm{B}$ (Fe and $\mathrm{Ni}$ ) and $e_{g^{-}}$ orbitals of $\mathrm{Ni}$ because the $\mathrm{B}-\mathrm{O}-\mathrm{Ni}$ angle is strictly $180^{\circ}$. The $t_{2 g^{-}}$-orbitals of transition metals are less involved in the exchange interaction. However, the finite overlap between these orbitals can be introduced by tuning the bond angles and lattice parameters as a consequence of mismatching the cations, thereby distorting the octahedra. The resultant transport properties suggest $\mathrm{Ba}_{2} \mathrm{FeNiO}_{6}$ heavily conduct like that of metal while $\mathrm{Ba}_{2} \mathrm{CoNiO}_{6}$ conductivity happens only after carries gain sufficient thermal energy. The ZT for $\mathrm{Ba}_{2} \mathrm{CoNiO}_{6}$ turns out to be $\sim 0.8$ at room temperature. The $100 \%$ spin polarization at the Fermi level indicates $\mathrm{FeNi}$-based perovskite can be used for spintronic applications and high $\mathrm{ZT}$ support $\mathrm{Ba}_{2} \mathrm{CoNiO}_{6}$ to be used as a thermoelectric material.

\section{Declarations}

\section{Conflict of Interest Statement:}

We hereby declare that we don't have any conflict of interest.

\section{Data Availability Statement:}

The datasets generated by the computation and thereafter analyzed would be available from Mr. Shabir Ahmad Mir on reasonable request.

\section{Author Contributions Statement}

Both authors have significant contributions to carry out this work: Shabir Ahmad Mir- conceptualization, methodology writing - original draft, revision, editing; Dinesh C. Gupta- supervision, software, validation, writing - review \& modification.

\section{References}

1. F. Geisz, R.M. France, K.L. Schulte, M.A. Steiner, A.G. Norman, H.L. Guthrey, M.R. Young, T. Song, T. Moriarty, Six-junction III-V solar cells with $47.1 \%$ conversion efficiency under 143 Suns concentration, Nature Energy 5,326-335 (2020).

2. Snider, N.D.-Gammon, R. McBride, M. Debessai, H. Vindana, K. Vencatasamy, K.V. Lawler, A. Salamat, R.P. Dias, Room-temperature superconductivity in a carbonaceous sulfur hydride Nature 586, 373-377 (2020).

3. H. Reshak, Spin-polarized Second Harmonic Generation from the Antiferromagnetic CaCoSO Single Crystal, Sci. Rep. 7, 1-7 (2017).

4. Bano, D.K. Pandey, A. Modi, N.K. Gaur, $\mathrm{MoB}_{2}$ driven metallic behaviour and interfacial charge transport mechanism in $\mathrm{MoS}_{2} / \mathrm{MoB}_{2}$ heterostructure: A first-principle study, Sci. Rep. 8, 1-11 (2018). 
5. A. Mir, D.C. Gupta. Systematic investigation of the magneto-electronic structure and optical properties of new halide double perovskites Cs2NaMCl6 ( $\mathrm{M}=\mathrm{Mn}$, Co and $\mathrm{Ni}$ ) by spin polarized calculations, RSC Adv 10, 26277-26287 (2020).

6. Byeon, R. Sobota, K.D. -Codrin, S. Choi, K. Hirata, M. Adachi, M. Kiyama, T. Matsuura, Y. Yamamoto, M. Matsunami, T. Takeuchi, Discovery of colossal Seebeck effect in metallic Cu2Se, Nature Communications10, 1-7 (2019).

7. Yousuf, D.C. Gupta, Thermoelectric response of ZrNiSn and ZrNiPb Half- Heuslers: applicability of semi-classical Boltzmann transport theory, Results Phys. 12, 1382 -1386 (2019).

8. M. Bhat, M. Nabi, D.C. Gupta, Electronic, elastic and thermoelectric performance in n-type Sr-filled brittle skutterudite, Physica B: Condensed Matter. 592, 1-6 (2020).

9. Mahmood, S.M. Ramay, W. Al-Masry, A. A. Al-Zahrani, N.Y.A. Al-Garadi, J. Mater. Res. Technol. 9, 14783-14791 (2020).

10. R. Pereda, N. Takeuchi, Density Functional Theory Study of the Organic Functionalization of Hydrogenated Graphene, J. Phys. Chem. C117, 18738-18745 (2013).

11. J. Yin, B. Weng, J. Ge, Q. Sun, Z. Li, Y. Yan, Oxide perovskites, double perovskites and derivatives for electrocatalysis, photocatalysis, and photovoltaics, Energy Environ. Sci.12, 442-462 (2019)

12. Vasala, M. Karppinen, $A_{2} B^{\prime} B^{\prime \prime} O_{6}$ perovskites: A review, Progress in Solid State Chemistry 43 (2015) 136

13. Kangsabanik, A. Alam, $A b$ InitioDiscovery of Stable Double Perovskite Oxides $\mathrm{Na}_{2} \mathrm{BIO}_{6}(\mathrm{~B}=\mathrm{Bi}$, In) with Promising Optoelectronic Properties, Phys. Chem. Lett. 11, 5148-5155 (2020)

14. Amores, H. El-Shinawi, I. McClelland, S.R. Yeandel, P.J. Baker, R.I. Smith, H.Y. Playford, P. Goddard, S.A. Corr, E. J. Cussen, $\mathrm{Li}_{1.5} \mathrm{La}_{1.5} \mathrm{MO}_{6}\left(M=\mathrm{W}^{6+}, \mathrm{Te}^{6+}\right)$ as a new series of lithium-rich double perovskites for all-solid-state lithium-ion batteries, Nat. Commun. 11, 1-12 (2020).

15. -I. Kobayashi, T. Kimura, Y. Tomioka, H. Sawada, K. Terakura, Room-temperature magnetoresistance in an oxide material with an ordered double-perovskite, Nature 395, 677-680 (1998).

16. Sahnoun, H. B. Benziane, M. Sahnoun and M. Driz, Magnetic and thermoelectric properties of ordered double perovskite $\mathrm{Ba}_{2} \mathrm{FeMoO}_{6}$, J. Alloys Compd. 714, 704-708 (2017).

17. -T. Jeng, G.Y. Guo, First-principles investigations of orbital magnetic moments and electronic structures of the double perovskites $\mathrm{Sr}_{2} \mathrm{FeMoO}_{6}, \mathrm{Sr}_{2} \mathrm{FeReO}_{6}$, and $\mathrm{Sr}_{2} \mathrm{CrWO}_{6}$, Phys. Rev. B: Condens. Matter Mater. Phys. 67, 1-7 (2003)

18. Jiang W.-J. Yin, High-throughput computational screening of oxide double perovskites for optoelectronic and photocatalysis applications, Journal of Energy Chemistry 57, 351-358 (2021)

19. Shen, Y. Jiang, S. Li, X. Lv, G. Yang, B. Sunden, Synthesis and experimental study of novel double perovskite $\mathrm{Ba}_{2} \mathrm{Ni}_{x} \mathrm{Co}_{2-\mathrm{x}} \mathrm{O}_{6}$ as promising oxygen carrier materials for $\mathrm{CO}_{2}$ capture application, Int $\mathrm{J}$ Energy Res. 44, 1-9 (2020)

20. Kumar, A. Kumar, Electrochemical behaviour of oxygen-deficient double perovskite, $\mathrm{Ba}_{2} \mathrm{FeCoO}_{6-\delta}$, synthesized by facile wet chemical process, Ceramics International 45, 14105-14110 (2019). 
21. A. Khandy, D.C. Gupta, Study of ferromagnetism, spin-polarization, thermoelectrics and thermodynamics of layered perovskite $\mathrm{Ba}_{2} \mathrm{FeMnO}_{6}$ under pressure and temperature, J. Phys. Chem. Solids, 135, December 1-7 (2019)

22. A. Dar, V. Srivastava, U.K. Sakalle, Structural, elastic, mechanical, electronic, magnetic, thermoelectric and thermodynamic investigation of half metallic double perovskite oxide $\mathrm{Sr}_{2} \mathrm{MnTaO}_{6}$, J. Magn. Magn. Mater. 484,298-306 (2019)

23. Nabi, T.M. Bhat, D.C. Gupta, Effect of pressure on electronic, magnetic, thermodynamic, and thermoelectric properties of tantalum-based double perovskites $\mathrm{Ba}_{2} \mathrm{MTaO}_{6}(\mathrm{M}=\mathrm{Mn}, \mathrm{Cr})$, Int. J. Energy Res. 43, 4229-4242 (2019).

24. A. Mir, D.C. Gupta, Understanding the origin of half-metallicity and thermophysical properties of ductile La2CuMnO6 double perovskite, Int. J. Energy Res. 43 (2019) 4783.

25. A. Mir and D. C. Gupta, Exploration of Uranium Double Perovskites $\mathrm{Ba}_{2} \mathrm{MUO6}(\mathrm{M}=\mathrm{Co}, \mathrm{Ni}$ ) for Magnetism, Spintronic and Thermoelectric Applications. Journal of Magnetism and Magnetic Materials, J. Magn. Magn. Mater. 493, 1-12 (2019).

26. Saha-Dasgupta, Double perovskites with $3 d$ and $4 d / 5 d$ transition metals: compounds with promises, Mater. Res. Express 7, 1-13 (2020).

27. Jana, P. Aich, P.A. Kumar, O.K. Forslund, E. Nocerino, V. Pomjakushin, M. Månsson, Y. Sassa , P. Svedlindh, O. Karis, V. Siruguri, S. Ray, Revisiting Goodenough-Kanamori rules in a new series of double perovskites $\mathrm{LaSr}_{1-x} \mathrm{Ca}_{x} \mathrm{NiReO}_{6}$, Sci. Rep. 9, 1-10 (2019)

28. Dabrowski, J. Mais, S. Kolesnik, O. Chmaissem, Parameters controlling magnetic interactions in perovskite manganites, J. Phys.: Conf. Ser. 303, 1-6 (2011).

29. M. Katukuri, P. Babkevich, O. Mustonen, H. C. Walker, B. Fåk, S. Vasala, M. Karppinen, H. M. Rønnow, O.V. Yazyev, Exchange Interactions Mediated by Nonmagnetic Cations in Double Perovskites, Physical Review Letters 124, 077202 (2020).

30. Birch, Finite Elastic Strain of Cubic Crystals, Phys. Rev. 71, 809-824 (1947).

31. D. Murnaghan, The Compressibility of Media under Extreme Pressures, Proc. Natl. Acad. Sci. U. S. A. 30, 244-247 (1944).

32. Nabi, D.C. Gupta, Study of the magneto-electronic, optical, thermal and thermoelectric applications of double perovskites $\mathrm{Ba}_{2} \mathrm{MTaO}_{6}(\mathrm{M}=\mathrm{Er}$, Tm), RSC Adv. 9, 15852-15857 (2019).

33. A. Sofi, D.C. Gupta, Investigation of structural, elastic, thermophysical, magneto-electronic, and transport properties of newly tailored Mn-based Heuslers: A density functional theory study, Int J Quantum Chem. 120, 1-19 (2020)

34. B. Yalcin, Band gap characterization of ternary $\mathrm{BBi}_{1-x} \mathrm{~N}_{x}(0 \leq x \leq 1)$ alloys using modified BeckeJohnson (mBJ) potential, Physica B: Physics of Condensed Matter 462, 64-69 (2015)

35. Jiang, Band gaps from the Tran-Blaha modified Becke-Johnson approach: A systematic investigation Journal of Chemical Physics 138, 1-7 (2013). 
36. Ozdogan, E. Sasıoglu, and I.Galanakis, Slater-Pauling behavior in LiMgPdSn-type multifunctional quaternary Heusler materials: Half-metallicity, spin-gapless and magnetic semiconductors, J.Appl.Phys. 113 193903-5 (2013).

37. Q. Seh, D. C. Gupta, Comprehensive DFT Investigation of Transition Metal Based New Quaternary Heusler Alloys CoNbMnZ (Z=Ge, Sn): Compatible for Spin Dependent and Thermoelectric Applications, RSC Adv. 10, 43870-43881(2020).

38. A. Mir, D.C. Gupta, Analysis of cage structured halide double perovskites Cs2 $\mathrm{NaMCl} 6(\mathrm{M}=\mathrm{Ti}, \mathrm{V})$ by spin polarized calculations, J. Alloys Compd. 854, 1-10 (2020).

39. J. DiSalvo, Thermoelectric Cooling and Power Generation, Science 285, 703-706 (1999).

40. P Blaha, K. Schwarz, Madsen GKH, Kvasnicka D, Luitz J. In: Schwarz K, ed. WIEN2K.An Augmented Plane Wave and Local Orbitals Program for Calculating Crystal Properties. Vienna: Vienna University of Technology; 2001.

41. Perdew, K. Burke and M. Ernzerhof, Generalized Gradient Approximation Made Simple, Phys. Rev. Lett. 77, 3865- 3868 (1996).

42. Koller, F. Tran, and P. Blaha, Improving the modified Becke-Johnson exchange potential, Phys. Rev. B 85, 155109-8 (2012).

43. K.H. Madsen and D.J. Singh"BoltzTraP. A code for calculating band-structure dependent quantities" Comput. Phys. Commun. 67, 175, (2006)

44. J. Scheidemantel, C. Ambrosch-Draxl, T. Thonhauser, J. V. Badding, and J. O. Sofo, Transport coefficients from first-principles calculations" Phys. Rev. B. 68, 125210 (2003).

45. Jodin, J. Tobola, P. Pecheur, H. Scherrer, and S. Kaprzyk, Effect of substitutions and defects in halfHeusler FeVSb studied by electron transport measurements and KKR-CPA electronic structure calculations, Phys. Rev. B. 70, 184207 (2003).

46. Otero-de-la-Roza, D. Abbasi-P'erez and V. Lua na, Gibbs2: a new version of the quasiharmonic model code. II. Models for solid-state thermodynamics, features and implementation, Comput. Phys. Commun. 182, 2232-2248 (2011)

\section{Figures}



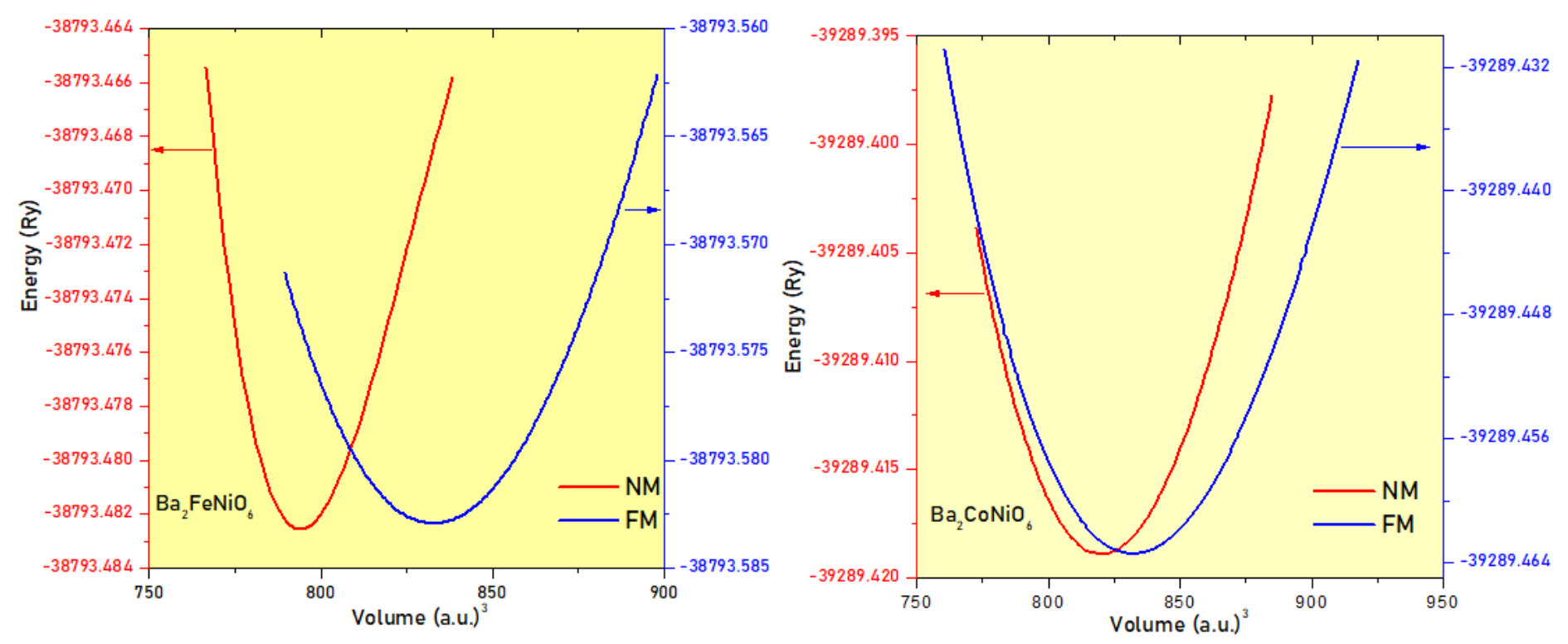

Figure 1

Total energy variation against volume for $\mathrm{Ba} 2 \mathrm{MNiO} 6(\mathrm{M}=\mathrm{Fe}, \mathrm{Co})$ double perovskites. The minima in the $\mathrm{E}-\mathrm{V}$ optimization curve correspond to the ground state energy, the equilibrium lattice parameters are evaluated corresponding to energy minima.
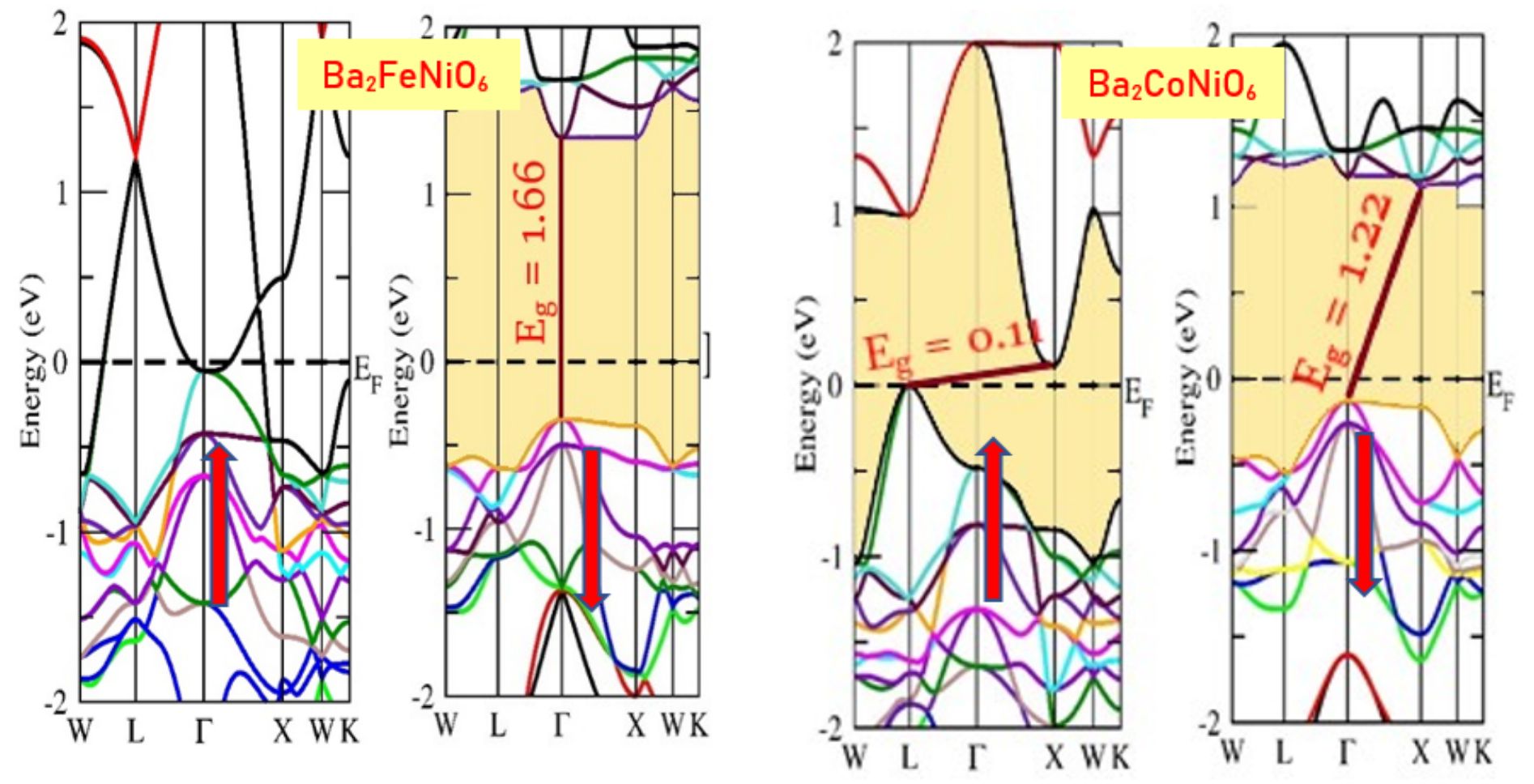

Figure 2

The spin-polarized band structure computed by employing GGA+mBJ approximation (the arrows are used to designate spin channels). 

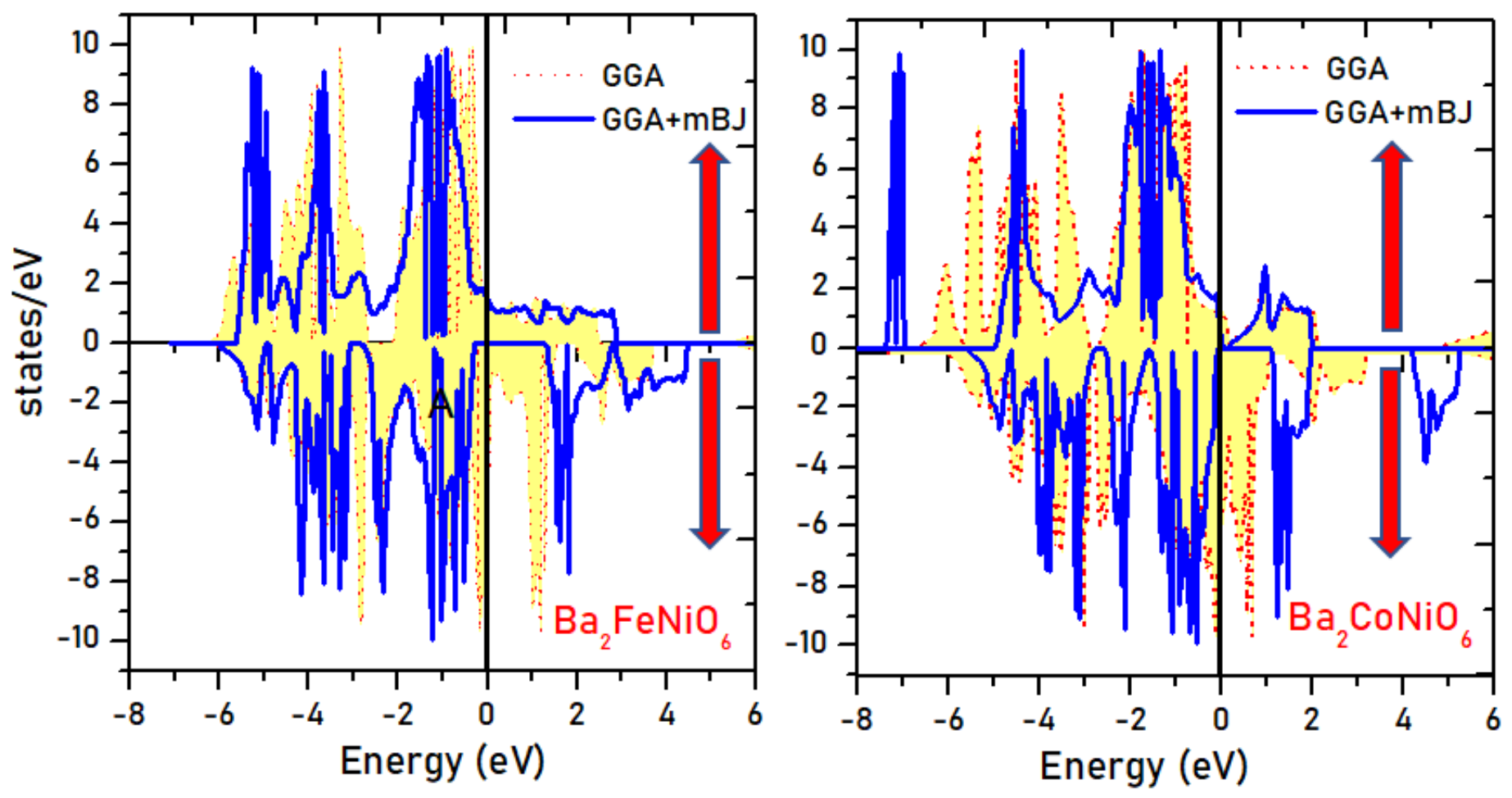

Figure 3

Effective variation in DOS distribution on integrating $\mathrm{mBJ}$ to GGA 


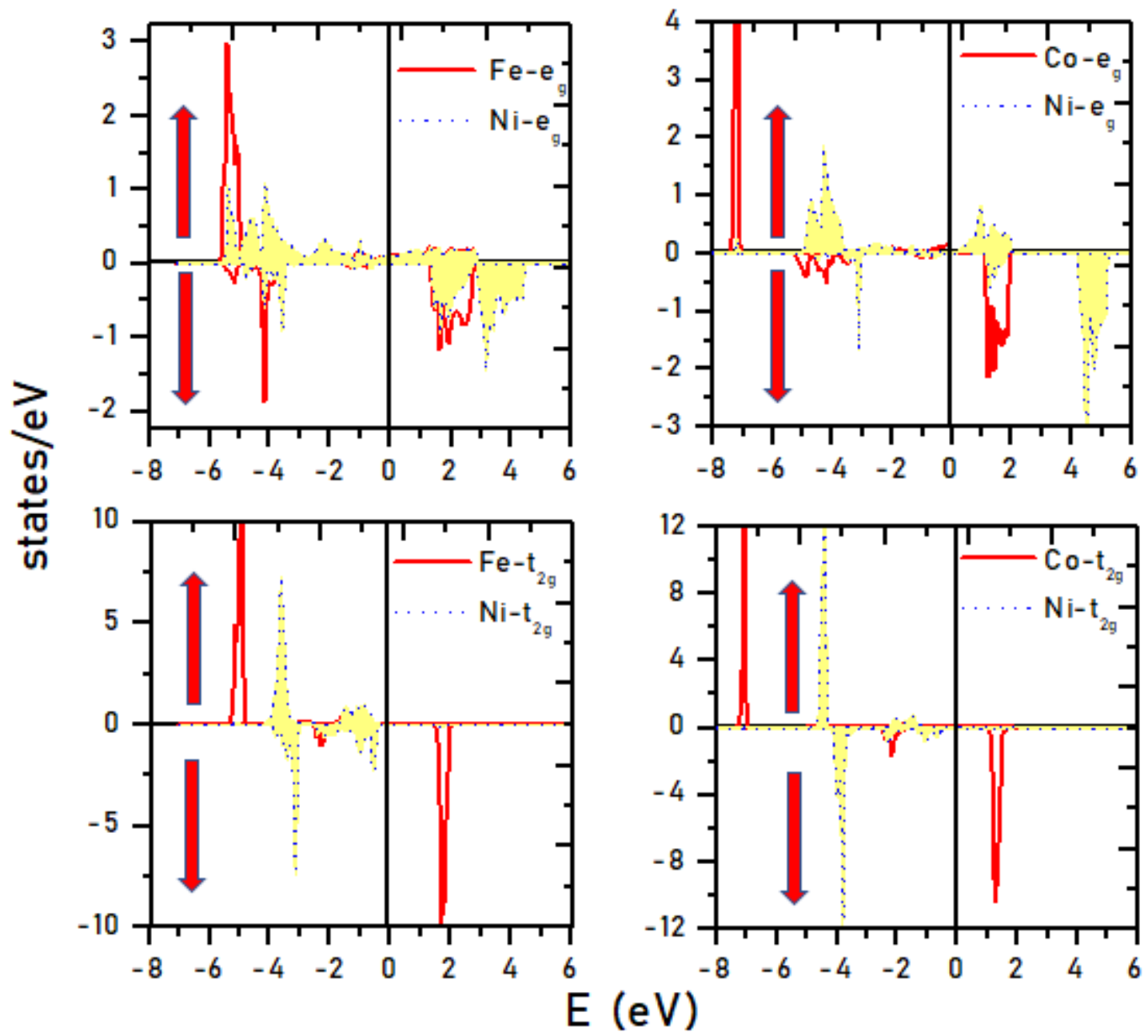

Figure 4

$\mathrm{t} 2 \mathrm{~g}$ and eg states distribution in $\mathrm{Ba} 2 \mathrm{MNiO} 6$ perovskites; eg-eg states are strongly hybridized while t2gstates of transition elements don't overlap reflect t2g-hybridization is ineffective 

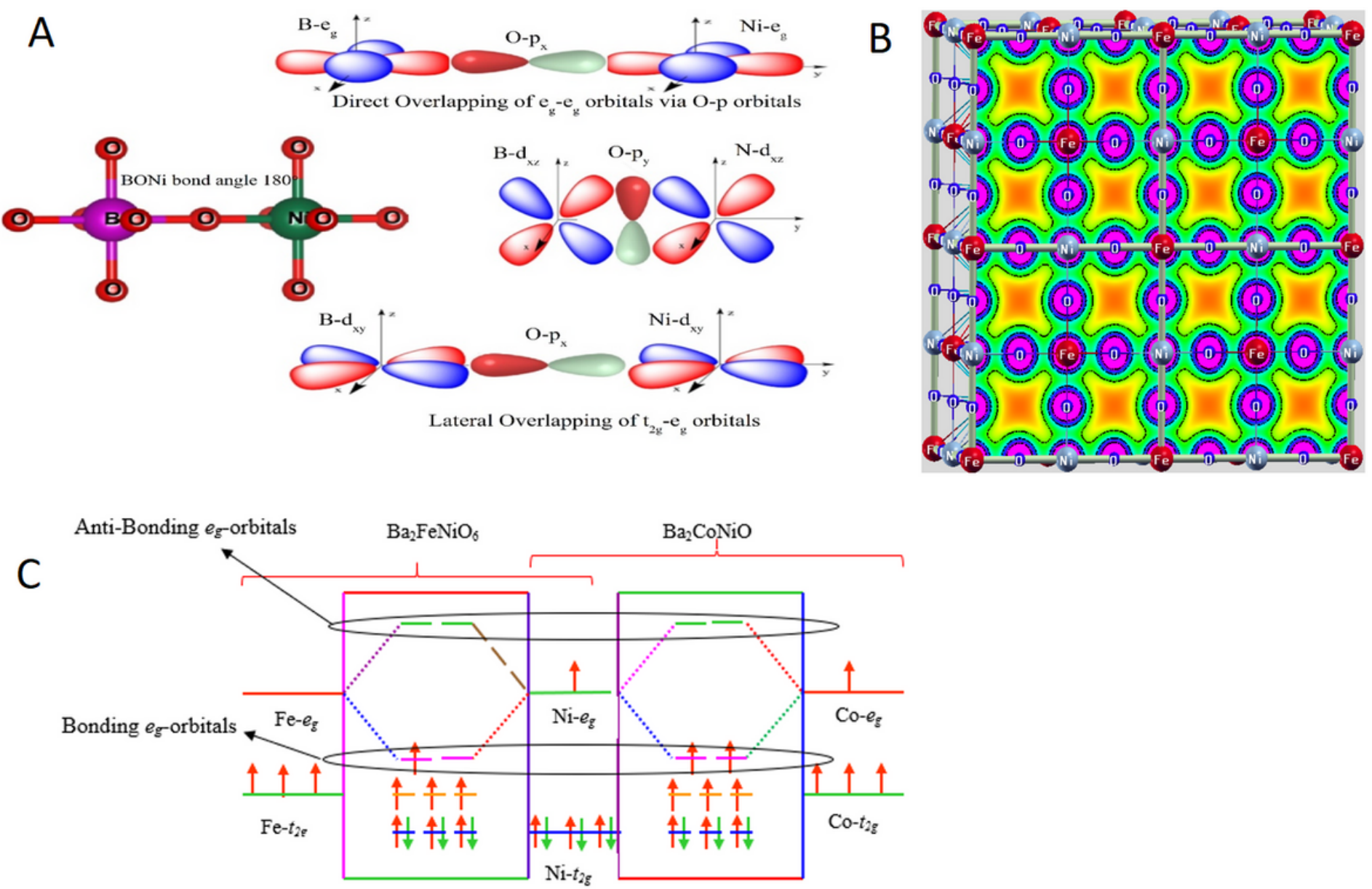

Figure 5

(a) Schematic representation of $d$-d hybridization among transition metals happening via oxygen $p$ states; (b) charge density distribution in <100> plane for Ba2FeNiO6. (c) Molecular orbital splitting and formation of hybridized bonding and anti-bonding states. 

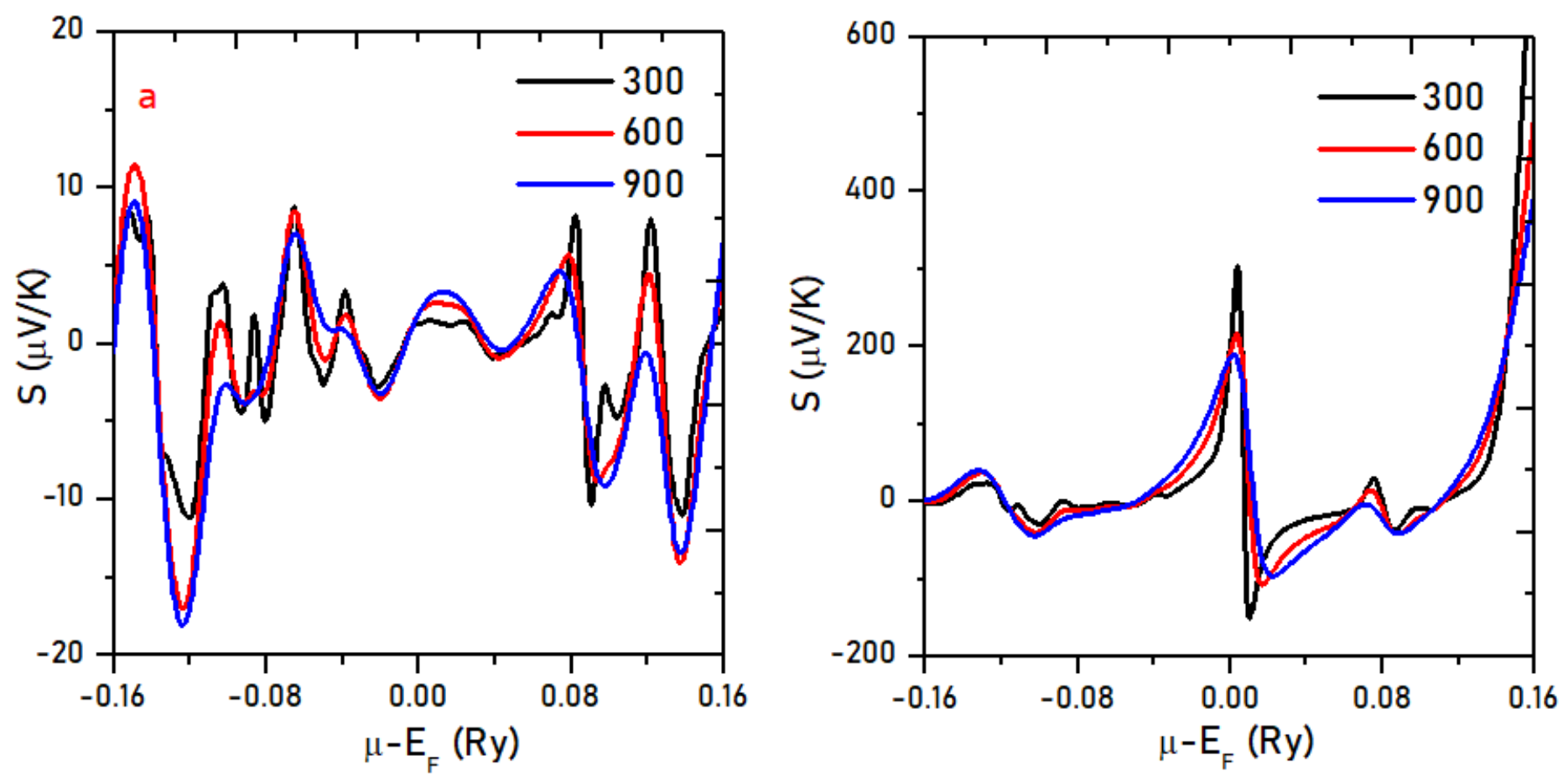

Figure 6

Total Seebeck coefficient as a function of chemical potential and temperature: (a) Ba2FeNiO6; (b) $\mathrm{Ba} 2 \mathrm{CoNiO6}$
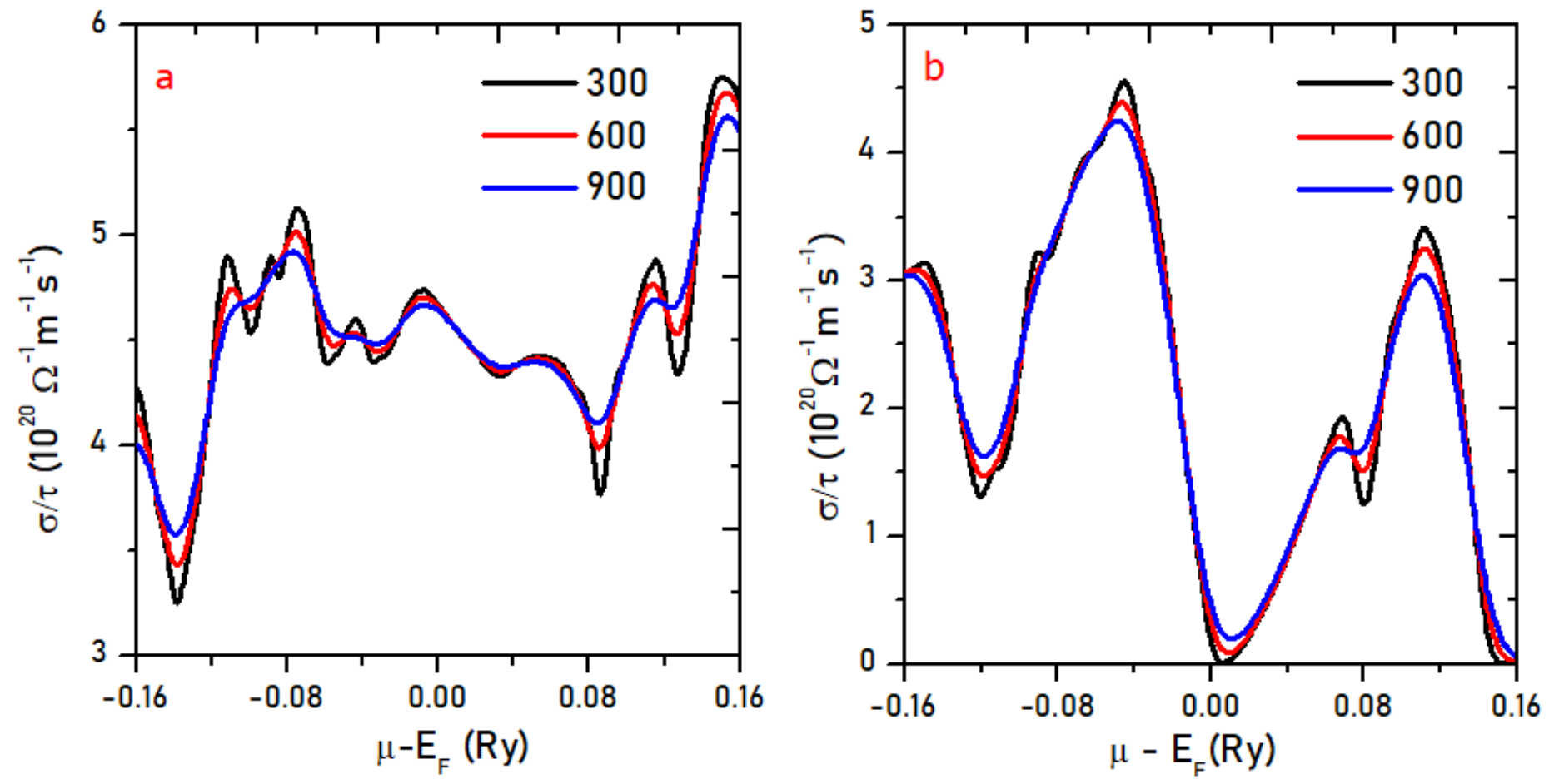

Figure 7 
Variation in total electronic conduction against chemical potential and temperature: (a) Ba2FeNiO6; (b) Ba2CoNiO6
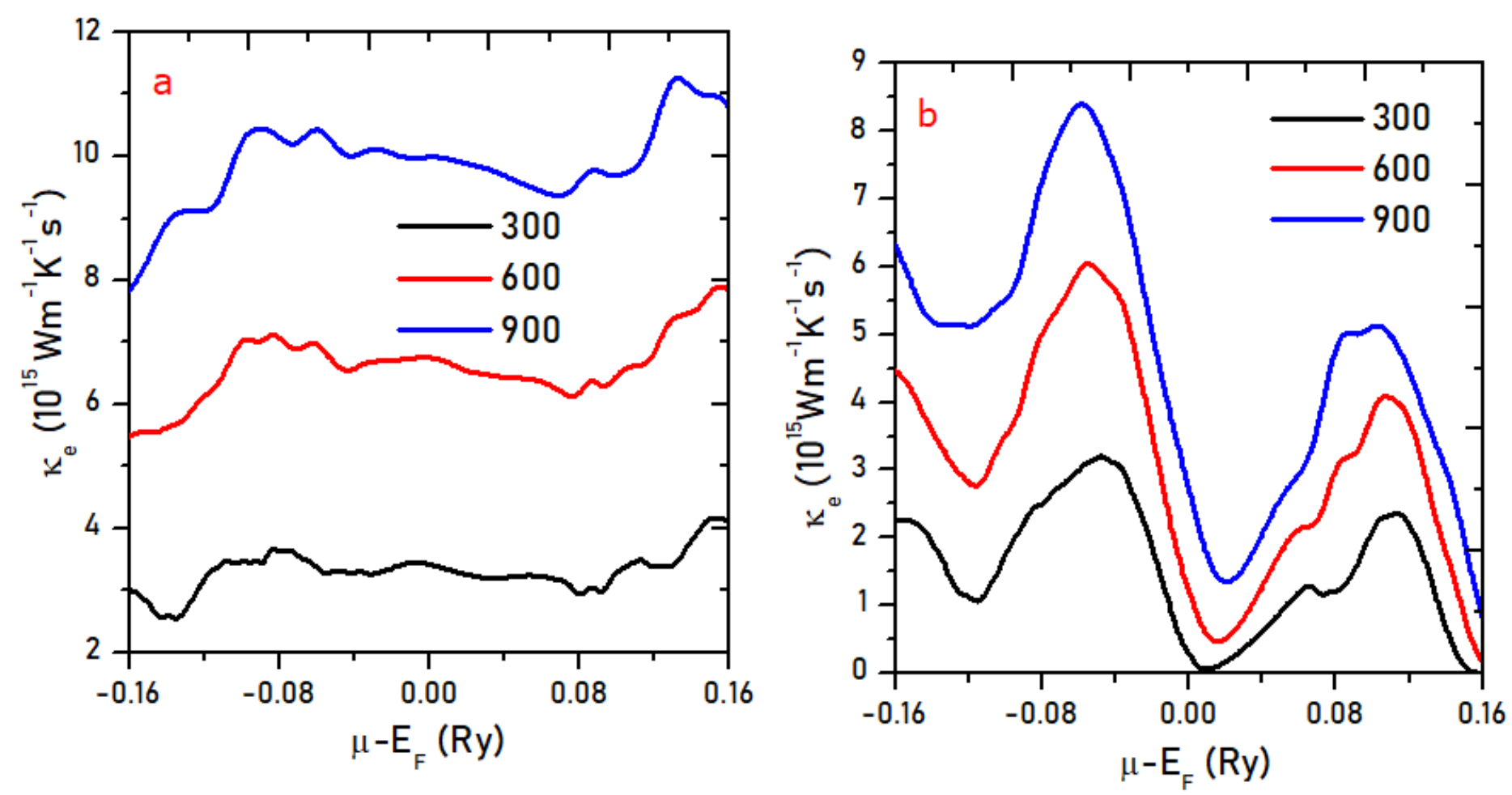

Figure 8

Variation in total electronic thermal conductivity with chemical potential and temperature: (a) Ba2FeNiO6;

(b) $\mathrm{Ba} 2 \mathrm{CoNiO6}$
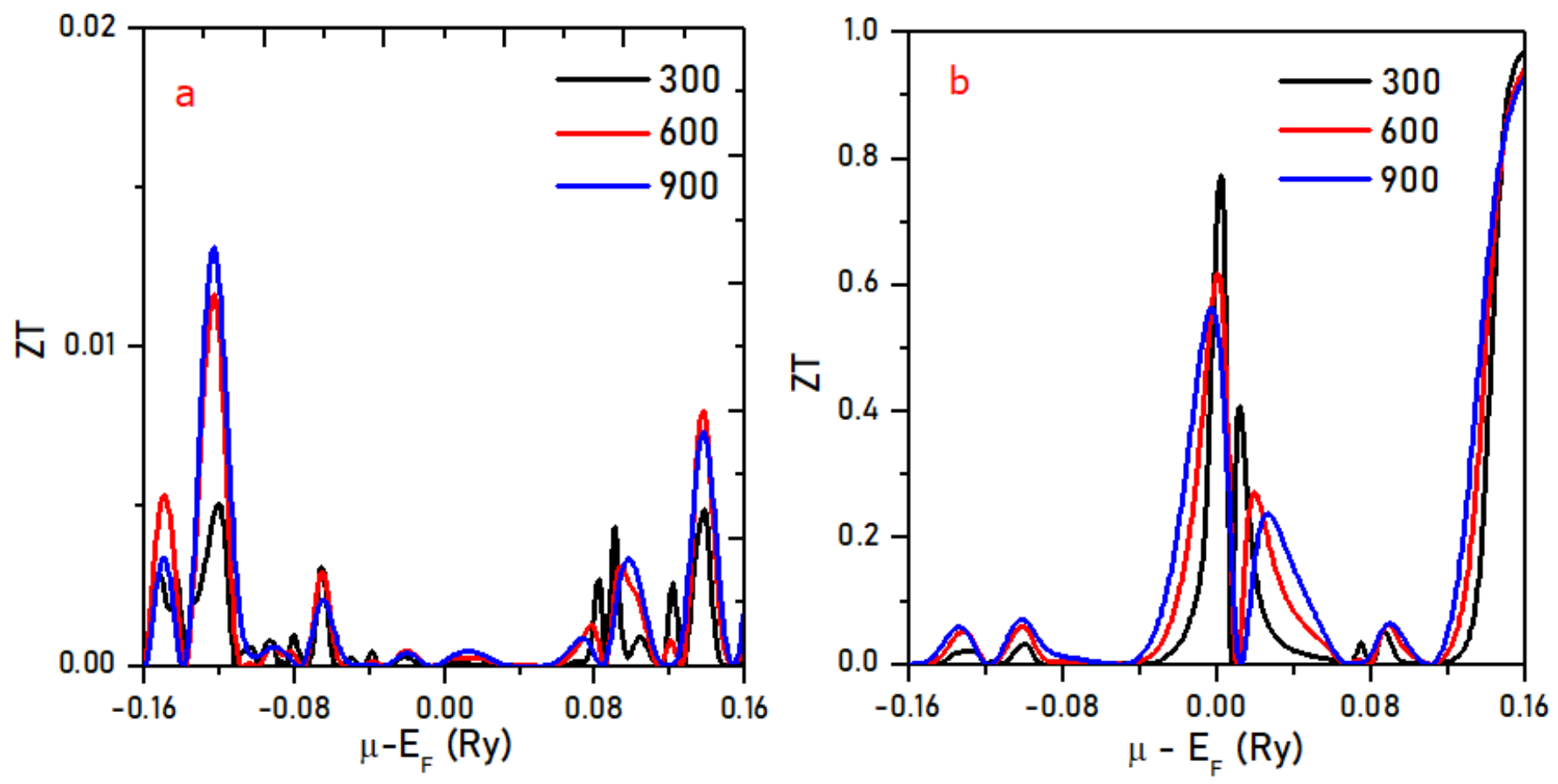


\section{Figure 9}

Behaviour of ZT without accounting lattice thermal conductivity with changing chemical potential at different temperatures: (a) Ba2FeNiO6; (b) Ba2CoNiO6

\section{Supplementary Files}

This is a list of supplementary files associated with this preprint. Click to download.

- Ba2MNiO6Supplementarylnformation.pdf 